\title{
A Mobile App for Thyroid Cancer Patients Aiming to Enhance Their Quality of Life: Protocol for a Quasiexperimental Interventional Pilot Study
}

Evanthia Giannoula ${ }^{1}, \mathrm{MSc}, \mathrm{MD}$; Ioannis Iakovou ${ }^{1}, \mathrm{MSc}, \mathrm{MD}, \mathrm{PhD}$; Ioannis Katsikavelas ${ }^{2}, \mathrm{MSc}$; Panagiotis Antoniou ${ }^{3}$, MSc; Vasilios Raftopoulos ${ }^{4}, \mathrm{MSc}, \mathrm{PhD}$; Vasiliki Chatzipavlidou ${ }^{5}, \mathrm{MSc}, \mathrm{MD}$; Nikitas Papadopoulos ${ }^{5}$, MSc, MD; Panagiotis Bamidis ${ }^{3}, \mathrm{MSc}, \mathrm{PhD}$

\footnotetext{
${ }^{1}$ Third Nuclear Medicine Department, Papageorgiou Hospital, Aristotle University of Thessaloniki, Thessaloniki, Greece

${ }^{2}$ Faculty of Engineering, Aristotle University of Thessaloniki, Thessaloniki, Greece

${ }^{3}$ Medical Physics Laboratory, Medical School, Aristotle University of Thessaloniki, Thessaloniki, Greece

${ }^{4}$ Department of Nursing, School of Health Sciences, Cyprus University of Technology, Limassol, Cyprus

${ }^{5}$ Nuclear Medicine Department, Theagenio Cancer Hospital, Thessaloniki, Greece
}

\section{Corresponding Author:}

Ioannis Iakovou, MSc, MD, PhD

Third Nuclear Medicine Department

Papageorgiou Hospital

Aristotle University of Thessaloniki

Periferiaki odos Thessaloniki

Thessaloniki,

Greece

Phone: 302313323506

Fax: 302310991491

Email: $\underline{\text { iiakovou@icloud.com }}$

\section{Abstract}

Background: Thyroid cancer (TC) is one of the fastest growing cancers all over the world. Differentiated thyroid cancer (DTC) is the most frequent subtype of TC. When appropriate treatment is given, the prognosis for the patient is generally excellent. Despite the generally good prognosis of thyroid carcinomas, the symptoms may range from emotional to physical discomfort, depending on the thyroid hormone status, which can severely affect the patient. Moreover, the diagnostic and therapeutic procedures that DTC patients have to undergo, such as thyroidectomy and radioiodine therapy, significantly affect their mental and physical well-being. Often, the physician only addresses the favorable prognosis of DTC compared with other cancer types and neglects to assess issues related to the quality of life (QoL) of the patient; this was the reason we decided to design a mobile app for DTC patients and their caregivers.

Objective: The aim of this study is to research the feasibility and applicability of an mHealth app tailored to DTC patients, as reflected in their QoL. The main features of the developed app offer access to useful information about thyroid cancer, diagnostic tests, and the appropriate therapy administered to DTC patients.

Methods: Based on the existing literature, we created an up-to-date information platform regarding TC and especially DTC. In order to develop an effective app that can be implemented in current health care, we designed a section where the patient and physician can keep a medical record in an effort to enable access to such information at any time. Finally, we designed a user-friendly notification program, including pill prescription, follow-up tests, and doctor visit reminders in order to equally facilitate the lives of the patient and physician.

Results: Having developed this mobile app, we aim to conduct a pilot quasiexperimental interventional trial. Our intention is to enroll at least $30 \mathrm{TC}$ patients and assign them to intervention or control groups. Both groups will receive standard care for treating and monitoring TC, and the intervention group will also receive and use the DTC app. TC patients' QoL will be assessed for both control and intervention groups in order to examine the effectiveness of the DTC app. QoL will be assessed through the QoL core questionnaire European Organisation for Research and Treatment of Cancer (EORTC) QLQ-THY34 in combination with the EORTC QLQ-C30 questionnaire through quantitative statistical analysis. 
Conclusions: The use of mHealth apps can play a significant role in patient education, disease self-management, remote monitoring of patients, and QoL improvement. However, the main limitation of the majority of existing studies has been the lack of assessing their usefulness as well as the absence of specific instruments to carry out this assessment. In light of those considerations, we developed a mobile app tailored to the needs of DTC patients. Furthermore, we evaluated its contribution to the QoL of the patients by using the EORTC QLQ-THY34 questionnaire, an accurate and safe instrument for the evaluation of the QoL in TC patients, while supporting future planned endeavors in the field.

International Registered Report Identifier (IRRID): PRR1-10.2196/13409

(JMIR Res Protoc 2020;9(3):e13409) doi: 10.2196/13409

\section{KEYWORDS}

mhealth; thyroid cancer; application; quality of life; patient-centered medicine; patient education

\section{Introduction}

Although thyroid cancer (TC) is a relatively rare neoplasm, accounting for approximately $1 \%$ to $5 \%$ of all cancers in females and less than $2 \%$ in males, it is the most common endocrine malignancy [1]. Furthermore, despite recent observations by Shi et al [2] that TC incidence rates for both sexes have declined since 2009, numerous other studies establish that thyroid gland neoplasms have shown the fastest increasing incidence of all malignancies over the past decades. This epidemiological trend, mostly attributed to overdiagnosis, has also been associated with several environmental factors [3]. According to the Surveillance, Epidemiology, and End Results Program of the National Cancer Institute, it is estimated that by the end of 2018 the number of new cases of TC will be 53,990 (3.1\% of all cancers), and approximately 2060 patients will die of the disease [4]. Differentiated thyroid cancer (DTC) is the most frequent subtype of TC and includes papillary and follicular types and their variants [5]. When appropriate treatment is given, the prognosis of the disease is generally excellent. Taking into consideration that DTC epidemiological trends and favorable prognosis present a high 5-year survival rate (up to 98.2\%) and an unimpaired life expectancy in most patients, it is crucial to ensure the quality of life (QoL) of the patient [6].

Surgery is the primary and most effective therapeutic approach for TC patients. Near total or total thyroidectomy with or without lymph node neck dissection is followed by radioiodine administration where appropriate. Thyroid stimulating hormone (TSH)-suppressive doses of thyroid hormone are administrated. This therapeutic management minimizes the risk of disease recurrence and metastatic spread while enabling accurate long-term surveillance. Systematic and targeted therapy is considered only for a small fragment of TC patients [7-10]. These therapeutic interventions, their preparation, possible complications or adverse events as well as the series of diagnostic and follow-up tests patients have to undergo may severely affect their QoL. TC patients often have difficulty in realizing the ways in which the disease will affect their everyday lives including, among other things, daily levothyroxine administration and frequent appointments with their doctors for diagnostic, therapeutic, and follow-up procedures. These procedures can be quiet stressful resulting in aggravating their mental health, especially in periods of hypothyroidism $[11,12]$. Moreover, the lack of proper information regarding the type of symptoms the patients will face, diagnostic and therapeutic procedures to which they are about to be submitted, and their impact on their physical and mental health accentuates feelings of isolation, fear, and anxiety [13,14]. Finally, caregivers play a crucial and demanding role regarding the care of TC patients, since they do not only exchange medical information with health care providers but also closely experience the disease of their loved ones to such an extent that they are often considered as a unit of care and may themselves experience mood disturbances and psychological impairment $[15,16]$.

Proper patient education is of great importance as it ensures that the patient understands the potential side effects of their treatment. A patient who is informed and aware of all the side effects of the respective treatment is likely to be more tolerant of the treatment than an uniformed one [17]. It is also crucial for them to understand the seriousness of the potential side effects in order to comply with physician recommendations to help prevent or minimize radiation-induced sequelae. Educational programs are required to adequately prepare not only patients but also health care professionals for future care. In particular, recognition of the totality of the cancer experience and the need for both staff and patient education were illustrated by Stajduhar et al [18]. If health care professionals were providing comprehensive cancer care, psychosocial and physical needs would be equally addressed. Fulfilling these needs requires a collaborative approach among patients and health care professionals, and more modern approaches such as mobile health (mHealth) are also of great assistance [18].

The use of mobile phones and mHealth apps is constantly proliferating [19]. The increasing use of health apps has been documented among health care professionals, younger and higher educated patients, and the general public. Apps can play a significant role in patient education, disease self-management, remote monitoring of patients, improving patient and caregivers QoL [20]. There have been many papers and reviews on the topic of mHealth. Even in cases of chronic diseases, such as cancer, patients expressed their preference regarding the use of apps. In a recent editorial for a relevant special issue, Coughlin [21] outlined the proliferation of mHealth by exploring institutional guidelines for smartphone development in the United States and other countries [22,23]. Previously, Bender et al [24] found 1314 potentially relevant apps, out of which 309 met the selection criteria for their systematic review on smartphone apps for the prevention, detection, and management of cancer. Eleven apps provided tools to support the management of cancer. The majority of these apps $(n=7)$ were 
not specific to a particular cancer type, and none of them was tailored for TC. These apps offered a combination of tools to assist the management of medical appointments, self-monitoring of symptoms, or medication consumption. The authors concluded that despite the existence of hundreds of cancer-focused apps, there is a lack of evidence regarding their utility, effectiveness, and safety [24]. Seiler et al [25] concluded, in a rather rigorous systematic review and meta-analysis, that very few mHealth apps targeted fatigued cancer survivors, however they revealed some rather interesting incidental results. First, most of the apps targeted healthy lifestyle maintenance, emphasizing dietary and physical activity goals; second, it was assessed that in most cases results were positive toward these goals [26-29]. Furthermore, in a systematic review/position paper, Nasi et al [30] succinctly outlined the mHealth environment. In short, they summarized the core feature that makes mHealth prolific, namely the flexibility of information availability in a wireless network. This was made apparent by the truism "a wireless network may be not mobile, but a mobile network must be wireless" [31]. Furthermore, this work managed to defined the key direction for mHealth apps which is not treatment itself but empowerment, information, and improvement in QoL for the patient [30]. In this study, it was also made clear that most of the literature in mHealth provided positive results and focused in chronic diseases and specifically cancer. More than a third of the surveyed literature (14/38, 37\%) comprised cancer subtopics such as chronic cardiovascular, pulmonary, and metabolic diseases (diabetes, asthma, obesity). Given adequately established interest in the field, a 4-dimensional framework for mHealth performance was introduced: efficiency, effectiveness, clinical effectiveness, and QoL [30]. Based on this framework, the aforementioned literature, and the fact that TC is a disease that is radically treated within a relatively short treatment time, our focus in this study was the fourth dimension, QoL. In this context, we designed and developed the first iteration of DTC, an mHealth app dedicated to DTC patients; in this study we aim to examine its effectiveness in patient QoL. Since the main limitation of most research is the lack of validated instruments for the evaluation of mHealth apps utility and effectiveness, the European Organisation for Research and Treatment of Cancer (EORTC) QLQ-THY34 questionnaire was used in order to yield accurate conclusions regarding the effectiveness of our intervention.

\section{Methods}

\section{Study Aims}

The primary objective of our study is to evaluate the design and implementation of an mHealth app tailored to DTC. This app aims to assist patients undergoing treatment for the management of malignant thyroid neoplasms by educating and motivating both them and their caregivers, facilitating maintenance of their medical record, and organizing their medication and appointments with their doctors. In order to examine the effectiveness of our intervention, we intend to conduct a quasiexperimental study assessing impact of the intervention on patient QoL as recorded in the EORTC QLQ-THY34 and EORTC QLQ-C30 questionnaires to reach accurate conclusions regarding the effectiveness of our app.

\section{App Development}

\section{Content of the App}

Our intention is to create an easy-to-use, accessible, and credible DTC app for patient QoL (Figure 1). We incorporated 4 different sections, with a specified function for each one of them. These included the core, informative Thyroid Info section which contains information regarding the thyroid gland and TC, especially DTC. The Add Patient and Patient Info sections enable both the doctor and patient to enter or view demographics and clinical data in order to keep an accessible medical record. Finally, the Add Reminder section includes an appointment organizer and pill reminder. The latter serves as an assistive tool to remind the patient to take daily levothyroxine and get properly prepared by undergoing thyroid hormone withdrawal or administering recombinant (rh)-TSH for diagnostic or therapeutic purposes. 
Figure 1. Screenshot of the DTC app home screen showing features Thyroid Info, Add Patient, Patient Info, and Add Reminder.

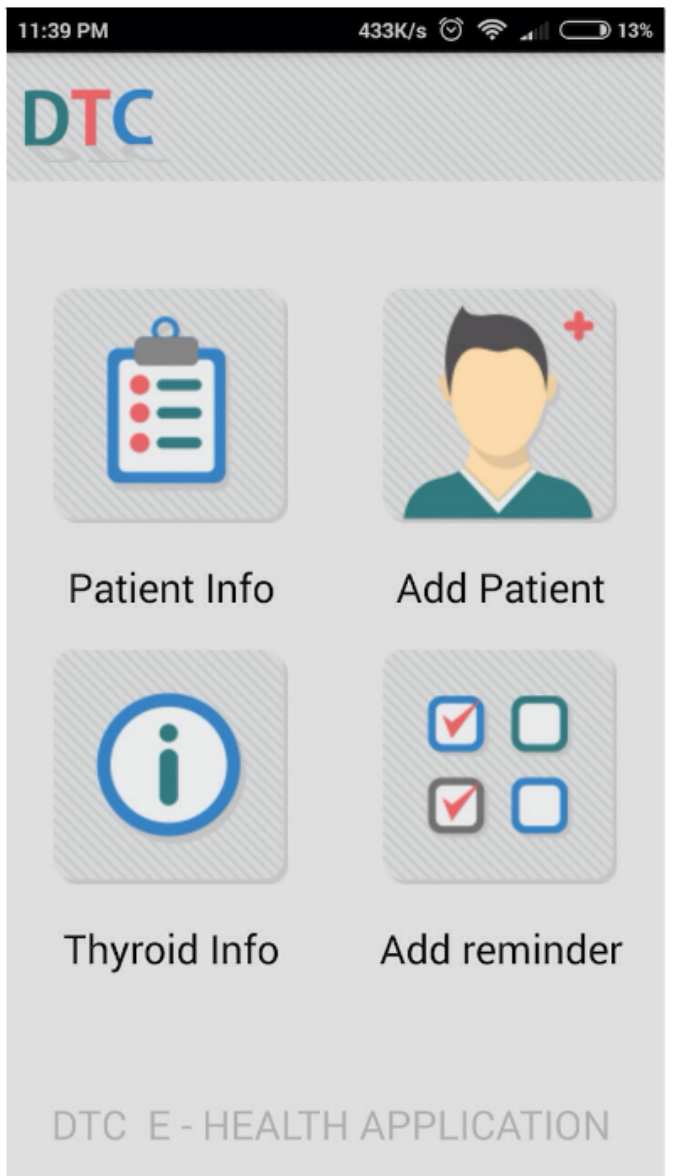

The importance of proper information and education on TC patients' effective disease management and therefore their prognosis, psychological status, and QoL is undisputed [17,18]. Given the amount of mostly nonvalidated and at times erroneous online information on thyroid malignancies, the majority of TC patients are rather misinformed and confused regarding their disease's progress and the diagnostic and therapeutic procedures they must undergo. In this context, we intend to create and provide an educational tool for our patients, available any time through their mobile device (phones, tablets, etc). After reviewing the literature, focused mainly on guidelines for the management of TC, we developed an up-to-date, accessible, and easily understood information section, Thyroid Info, in clear and plain language (Figures 2 to 4). We conducted a literature search between September 2015 and July 2016 to review all aspects of thyroid function and neoplasms and their management from diagnosis to treatment and follow-up using the following electronic databases: PubMed, Highwire, and Google Scholar. The keywords and terms used in the literature research were: "hormones," "thyroid gland," "thyroid cancer," "epidemiology," "risk factors," "management," "diagnosis," "treatment," "radioiodine," "adverse effects," "guidelines," "TC," and "pregnancy." These terms were used individually and in combination with the following links: or, and. Additional articles and reports were accessed via citations in reviewed papers that appeared in the original research. Our final database contains 71 publications. Our app is directed toward Greek patients, therefore we chose to use Greek in the app's text. Thyroid Info consists of chapters on hormones and endocrine glands, thyroid gland, thyroid gland nodules, malignant neoplasms of the thyroid gland, management of TC, radioiodine treatment of DTC, follow-up of TC, TC and pregnancy, and the references we used. We structured Thyroid Info taking into consideration the respective literature and the disease issues that affect our patients. Each chapter is divided into several questions, allowing the patient to select the question that best matches their needs. Our text contains figures and tables rendering access to information easier and more targeted. 
Figure 2. Thyroid Info feature comprises 8 chapters and references. Each chapter contains several questions so the patient can have the option to choose the topic they are interested in each time.

\begin{tabular}{|c|}
\hline $431 \mathrm{~K} / \mathrm{s}$ ह $>4 \subset 12 \mathrm{~s}$ \\
\hline 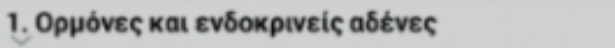 \\
\hline 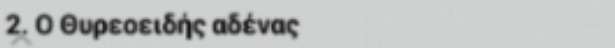 \\
\hline 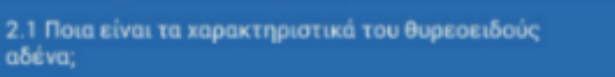 \\
\hline 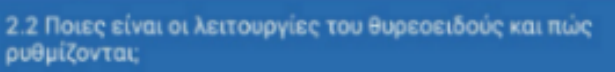 \\
\hline 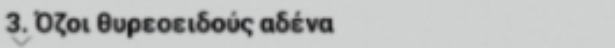 \\
\hline 4. Какоп் \\
\hline 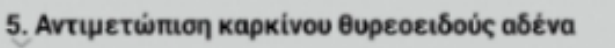 \\
\hline 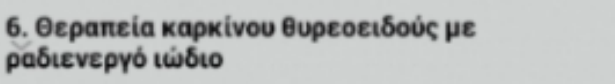 \\
\hline 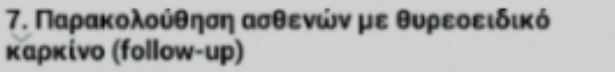 \\
\hline 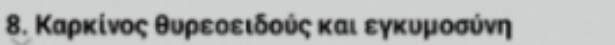 \\
\hline
\end{tabular}

Figure 3. Screenshot of Thyroid Info feature content.

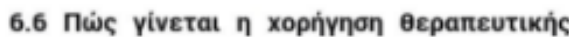

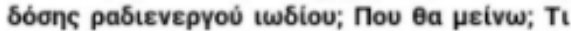

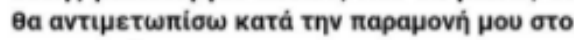
vобоконвіо;

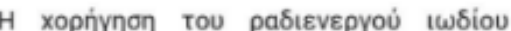

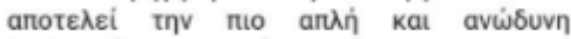

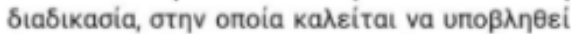

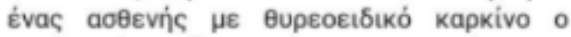

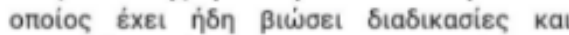

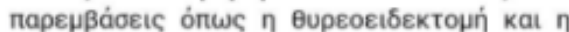

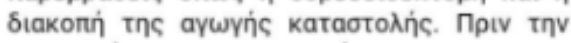
Eเøaywyń tou kat tn xopriynon tou 1311, o

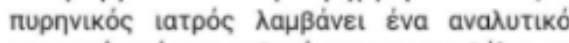

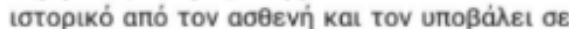

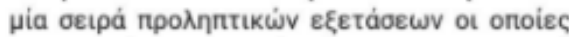

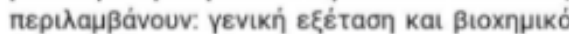
$\dot{\varepsilon} \lambda \varepsilon ү x о$ aipatoc, $\dot{\varepsilon} \lambda \varepsilon \gamma x о$ TwV TSH, TG, TgAb.

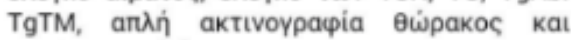

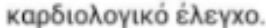

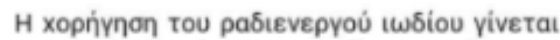

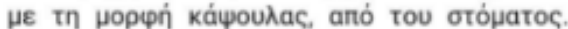

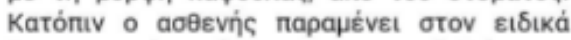

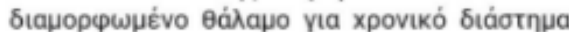

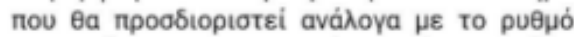

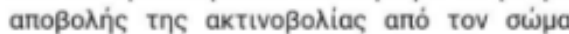

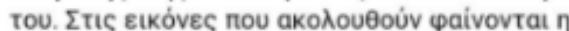

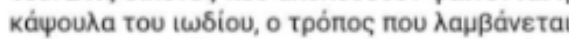

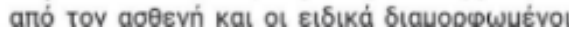


Figure 4. Screenshot of Thyroid Info feature content.

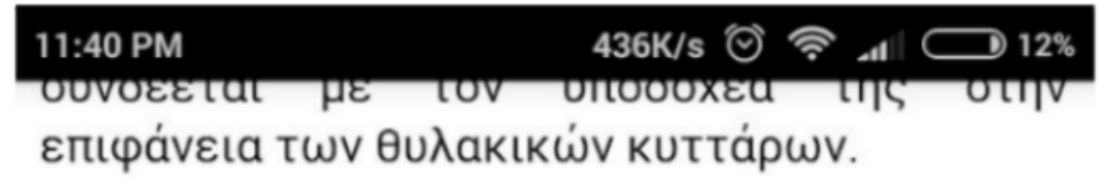

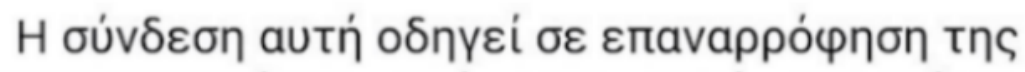

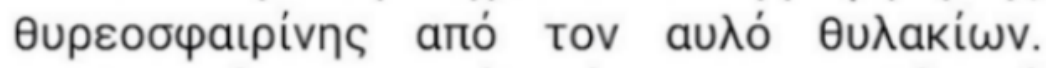

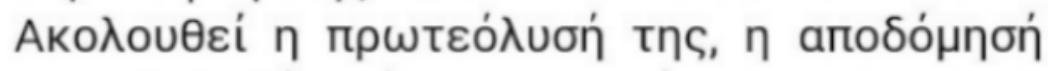

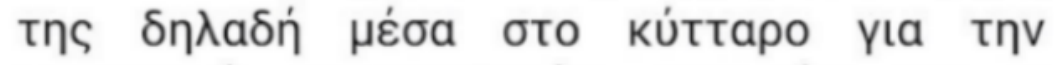

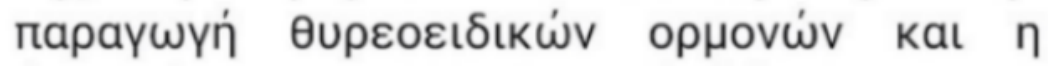

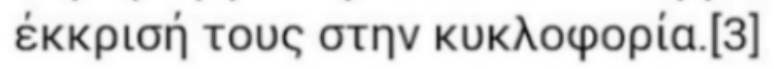

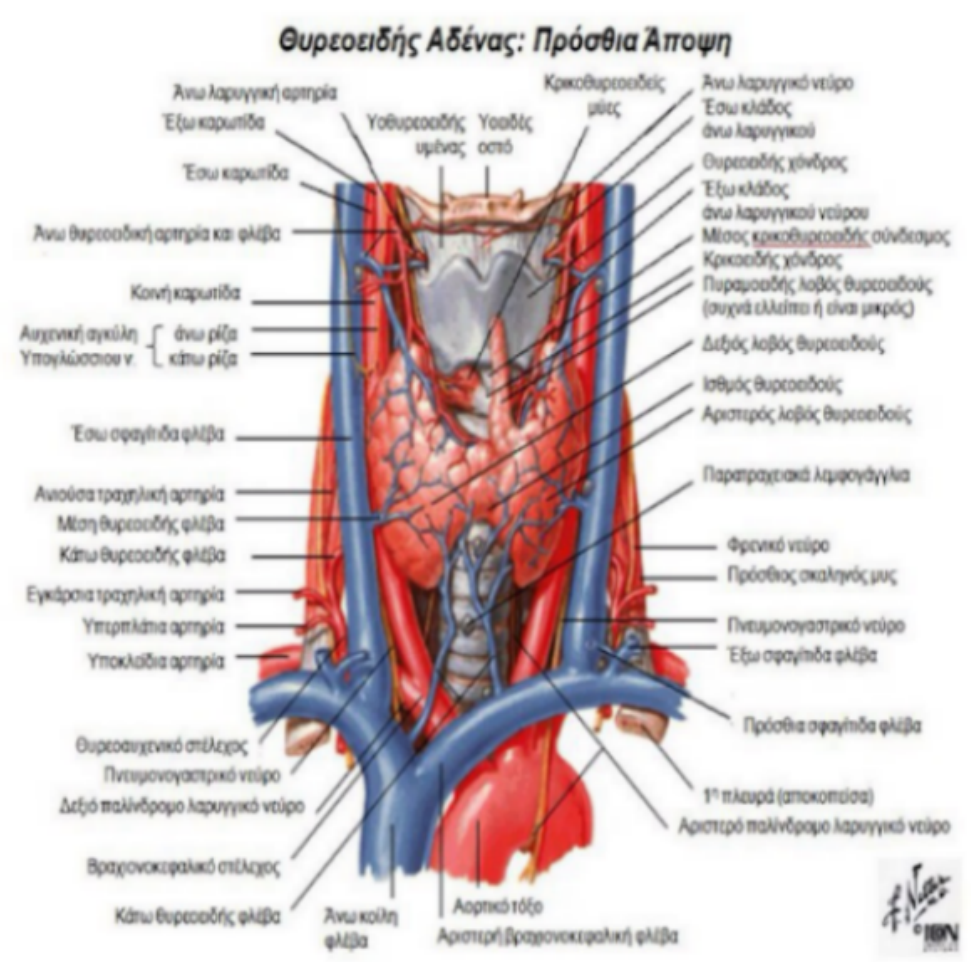

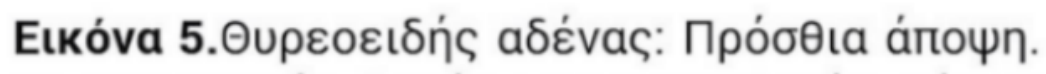

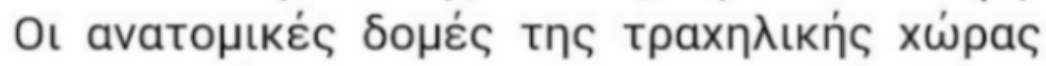

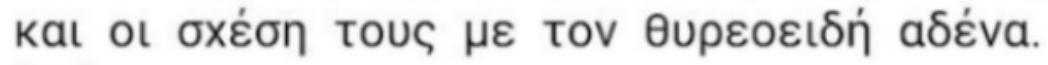 [11].}

In the Add Patient panel, the patient or physician can add relevant demographic and clinical information and the results of any follow-up tests (Figure 5). Information includes registration number, name, surname, username, password, email, code, phone number, address, gender, date of birth, age at diagnosis, date of surgery, name of surgeon, histology, maximum diameter of the largest TC foci, number of foci, thyroid gland lobe that TC was detected, invasion, number of excised lymph nodes, number of excised lymph nodes with metastases, stage, date of postsurgical remnant thyroid gland scintigraphy, date of radioiodine ablation, days between surgery and radioiodine ablation, preparation (thyroid hormone withdrawal or rh-TSH administration), I-131 scintigraphy, 24 hour uptake of I-131, neck-thyroid bed ultrasound, and levels of TSH, thyroglobulin, thyroglobulin antibodies, and thyroperoxidase antibodies. The Patient Info platform will serve as a medical record, containing patients' registered data, available with the use of username and password set by the patient (Figure 5). The doctor or administrator has the option of adding new patients and their data from their personal computer. 
Figure 5. Screenshot of an Add Patient page. Registration can be done either in English or Greek.

\section{GR COSMOTE 可 0.95K/s ร..11 回18:30}

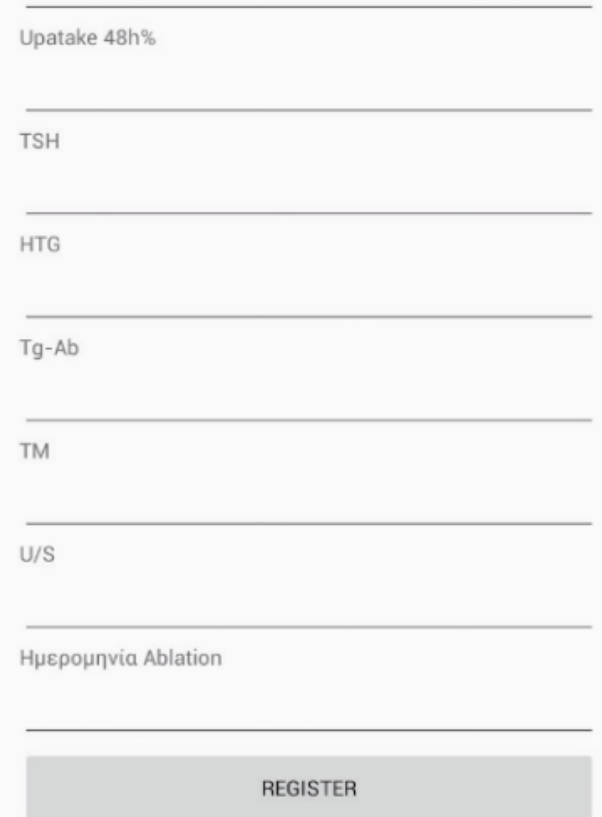

The Add Reminder panel includes appointment organizer or reminder. This section was designed to serve as an appointment reminder and levothyroxine administration alert, enabling proper preparation for diagnostic and therapeutic procedures. The reminder will inform patient regarding when to withdraw thyroid hormone medication or rh-TSH injections in order to receive radioiodine for diagnostic or therapeutic purposes. Given that the volume of notifications received by users can be significant, we decided to use only a small number of notifications. When a patient visits the doctor, they can jointly arrange the next visit and prescribed medication and store this information in the app. In this context, we used Google's Calendar API (application programming interface) to enable an organized display of the patient's appointments and medication plans. The patient receives notifications for appointments a few days before the appointment and on the very day of the appointment. Regarding the medications, notifications are also used as a reminder. To best accommodate the needs of each patient, notifications can be edited.

\section{App Design}

The app was designed and developed in the Android Studio Development environment. The architecture used in this specific research study is a basic mobile architecture based on easy-to-use interface, system security, and data integrity. The app is easily deployable on any Android mobile platform, and plans are underway for the development of an iOS app. This app aims to provide a simple and intuitive interface, access to medical information (written by specialist doctors), and the ability to enable, store, and retrieve patient data (demographic and medical) in and from the cloud. The app also provides information to the user through notifications.

It is well known that the health care sector is extremely sensitive and has specific data protection requirements. Designing and developing a mobile app that contains sensitive patient data requires adherence to strict rules necessary to prevent unauthorized access to private information or personal data on the patient. It is also important to maintain updated log records for system failures. In view of the above, security has been one of our major concerns when designing and developing the DTC app. Data confidentiality and integrity has been ensured, while user and app authentication have been verified. This is the reason why we opted for cloud storage. Every data structure we store to the cloud is cryptographed. Tools such as hash functions, digital signatures, and symmetric and asymmetric keys are needed to achieve these features. Apart from that, we work on integrating the HL7 standards for the transfer of clinical and administrative data between software apps used by various health care providers.

Generally, the app design was geared to support the patient during treatment and management of DTC by informing and educating them. Furthermore, it was designed to empower them by keeping an up-to-date and easily accessible medical record while respecting their privacy, and providing reminders for medication and doctor appointments. Overall, the DTC mHealth app aims to become a key component in contributing to the improvement of patient QoL. 


\section{Results}

\section{Study Design}

For this interventional study, we chose a quasiexperimental design to examine DTC patients' QoL. The quasiexperimental approach was selected instead of the more rigorous randomized controlled trial (RCT) for two reasons. The first was the exploratory nature of this research since it is aiming to scope the features of such an app specifically pertaining to TC. The second is the necessity for credible but rapid results in order to iterate on the app itself. An RCT type of study is planned for a future iteration of the DTC app.

The main weaknesses of most of the previous studies regarding mHealth are the lack of assessment of the feasibility, usefulness, and effectiveness of mHealth apps and the lack of specific instruments for this assessment. As far as thyroid-related problems and functioning are concerned, only a few thyroid-specific QoL questionnaires have been developed. Moreover, we are informed assessments have been done largely using generic instruments. The EORTC created a TC-specific module to be used as a supplement to the QoL core questionnaire EORTC QLQ-C30 (phase I and II of the EORTC module development process) [32,33]. In these previous phases, items were derived from a longer list of QoL issues that could be relevant for TC patients. Phase III of the instrument development, according to the EORTC module development guidelines published in 2017, resulted in the EORTC QoL module for TC (EORTC QLQ-THY34) currently at the final phase IV validation which will be used in our study [34]. We acknowledge that using the not-yet-validated version of the EORTC THY34 is both a strong and a weak point of our study. By using the EORTC QLQ-THY34 questionnaire in combination with EORTC QLQ-C30 questionnaire, our intention is to reach safe and accurate conclusions given that both the app and questionnaire are tailored for TC [35].

\section{Participant Recruitment}

Patients will be recruited at the 3rd nuclear medicine department of Papageorgiou Hospital, Aristotle University of Thessaloniki, Greece, and the nuclear medicine department of Theagenio Cancer Hospital, Thessaloniki, Greece. A formal protocol of the study has been submitted and approved by the ethics committee of the Aristotle University of Thessaloniki (no 398/11.12.2017) that shall include written consent from the patients to use their pseudonymized data in a statistically aggregate manner while respecting their privacy. We aim to enroll at least 30 patients into two groups without randomization. The first group will be given the DTC app, while the rest of the patients will receive oral instructions and informative printed material. Both groups will undergo standard diagnostic and therapeutic procedures the DTC patients receive. The inclusion criteria for patient participation in the intervention group are having undergone thyroidectomy, diagnosis of DTC, radioiodine treatment medically indicated, possession of mobile phone or tablet supporting Android software, and the ability to use Android apps. The control group will have similar demographic and clinical characteristics.

\section{Data Collection}

For both groups there will be recorded demographic and clinical characteristics. As far as the intervention group is concerned, the data will be recorded by the nuclear medicine physician either via the DTC app on the patient's mobile device or the physician's personal computer. Following that, data will be exported in Excel (Microsoft Corp) files and stored in SPSS Statistics 25.0.0.0 (IBM Corp). Control group data will be recorded in Excel files and then stored in SPSS Statistics 25.0.0.0. Patients of both groups will be given the EORTC QLQ-THY34 and EORTC QLQ-C30 questionnaires to examine their QoL. This is the only instrument in the native language of the patients, developed by the EORTC QoL Group. The EORTC QoL module for TC consists of 34 items and is currently being pilot-tested for the final international field validation in phase IV. It will be available in the following languages: Arabic, Chinese, Dutch, English, French, German, Greek, Hebrew, Hindi, Japanese, Italian, Polish, Portuguese, and Tamil [32]. The module has been developed according to the EORTC guidelines and approved after formal review. The EORTC QLQ-THY34 is still under development and is thus being shared only with those groups willing to provide data relevant to evaluating its psychometric properties. In this framework, we received authorization to use the EORTC QLQ-THY34 questionnaire, assuring our compliance with the EORTC's rules. Considering that the EORTC QLQ-THY34 questionnaire is formally translated and validated in Greek, it is appropriate to argue that it constitutes the most accurate and safe instrument to evaluate QoL in TC patients, thus ensuring the validity of our results on the effectiveness of the DTC app.

\section{Data Analysis}

All data will be downloaded and stored in SPSS Statistics. Descriptive statistics will be used to characterize the overall sample, each condition, and study acceptability and demand. To examine preliminary differences in feasibility, we will use chi-square tests, Fisher exact tests, and nonparametric Mann-Whitney $U$ tests for nonnormal data at the level of significance of $P<.05$ as appropriate.

Clinical evolution questions and diagnostic markers shall be checked for possible psychological effects of better information (eg, question 34: Have you experienced any throat ache?). However, the respective analysis shall focus on questions describing both the emotional disposition of the patient (eg, Did you feel distressed or irritated?) and their perceptions about the quality of care provided (eg, Did you feel well supported by your doctors?). The hypothesized scales assessed for our patients through the questionnaires are anxiety, body image, cramps, discomfort in the head and neck, dry mouth, fatigue, hair, impact on job or education, joint paint, neuropathological problems, restlessness, shoulder functioning, social support, swallowing, temperature tolerance, voice, and worries about important others. 


\section{Discussion}

\section{Mobile Health in the Service of Thyroid Cancer Patients}

TC is a malignant neoplasm with excellent prognosis in cases where appropriate treatment is given. The standard therapeutic interventions, their preparation, possible complications or adverse events, and numerous diagnostic and follow-up tests that TC patients must undergo may severely affect their QoL. Evidence-based medicine, a more personalized patient approach, novel management strategies such as the use of rh-TSH, and proper patient education are intended to ensure QoL. The use of mHealth apps — currently proliferating — can play a significant role in patient education, disease self-management, and remote monitoring of patients, thus resulting in an improvement in their QoL. There have been already several papers and reviews on the topic of mHealth addressed in conjunction with chronic diseases and, more specifically, with cancer. However, the key weakness of most of the studies to date has been the lack of assessing their usefulness and specific instruments used in the assessment. In this context, we attempted to develop a mobile app tailored to DTC patients and evaluate its contribution to patient QoL by using the EORTC QLQ-THY34 questionnaire. To the best of our knowledge, the QLQ-THY34 questionnaire is the most accurate and safe instrument to evaluate QoL in TC patients and is also under validation and translated in Greek, which ensures the validity of our results on the effectiveness of the DTC app.

The work described here is a precursor to a planned RCT endeavor on the impact of such solutions on the overall QoL aspect of cancer survivorship, an active and open research endeavor. This work aims to assess how effective the app is regarding the improvement of QoL of these patients (soon to be considered as productive and healthy individuals), evaluate the DTC mHealth solution regarding the patient's access to medical information, and provide moral support during and after treatment. This shall be achieved with validated evaluation instruments through accepted common practices at two of the largest hospitals in Greece. This quasiexperimental study shall provide evidence-based feedback for the iterative refinement of this mHealth solution and support future planned endeavors and research in the field.

\section{Acknowledgments}

We would like to express our gratitude to the Medical Physics Department of Aristotle University of Thessaloniki, Greece, for its financial contribution for covering the publishing fee.

\section{Conflicts of Interest}

None declared.

\section{References}

1. Kilfoy BA, Zheng T, Holford TR, Han X, Ward MH, Sjodin A, et al. International patterns and trends in thyroid cancer incidence, 1973-2002. Cancer Causes Control 2009 Jul;20(5):525-531 [FREE Full text] [doi: 10.1007/s10552-008-9260-4] [Medline: 19016336$]$

2. Shi LL, DeSantis C, Jemal A, Chen AY. Changes in thyroid cancer incidence, post-2009 American Thyroid Association guidelines. Laryngoscope 2017 Dec;127(10):2437-2441. [doi: 10.1002/lary.26473] [Medline: 28092116]

3. Giannoula E, Iakovou I, Chatzipavlidou V. [Risk factors and the progression of thyroid malignancies]. Hell J Nucl Med 2015;18(3):275-284 [FREE Full text] [doi: 10.1967/s002449910307] [Medline: 26574695]

4. National Cancer Institute. Cancer stat facts: thyroid cancer URL: https://seer.cancer.gov/statfacts/html/thyro.html [accessed 2017-12-29] [WebCite Cache ID 6w4GQn6nS]

5. Tunca F, Sormaz IC, Iscan Y, Senyurek YG, Terzioglu T. Comparison of histopathological features and prognosis of classical and follicular variant papillary thyroid carcinoma. J Endocrinol Invest 2015 Dec;38(12):1327-1334. [doi: 10.1007/s40618-015-0376-6] [Medline: 26280320]

6. Verburg FA, Mäder U, Tanase K, Thies E, Diessl S, Buck AK, et al. Life expectancy is reduced in differentiated thyroid cancer patients $\geq 45$ years old with extensive local tumor invasion, lateral lymph node, or distant metastases at diagnosis and normal in all other DTC patients. J Clin Endocrinol Metab 2013 Jan;98(1):172-180. [doi: 10.1210/jc.2012-2458] [Medline: 23150687]

7. American Thyroid Association (ATA) Guidelines Taskforce on Thyroid Nodules and Differentiated Thyroid Cancer, Cooper DS, Doherty GM, Haugen BR, Hauger BR, Kloos RT, et al. Revised American Thyroid Association management guidelines for patients with thyroid nodules and differentiated thyroid cancer. Thyroid 2009 Nov;19(11):1167-1214. [doi: 10.1089/thy.2009.0110] [Medline: 19860577]

8. Luster M, Clarke SE, Dietlein M, Lassmann M, Lind P, Oyen WJG, European Association of Nuclear Medicine (EANM). Guidelines for radioiodine therapy of differentiated thyroid cancer. Eur J Nucl Med Mol Imaging 2008 Oct;35(10):1941-1959. [doi: 10.1007/s00259-008-0883-1] [Medline: 18670773]

9. Haugen BR, Alexander EK, Bible KC, Doherty GM, Mandel SJ, Nikiforov YE, et al. 2015 American Thyroid Association management guidelines for adult patients with thyroid nodules and differentiated thyroid cancer: the American Thyroid Association Guidelines Task Force on thyroid nodules and differentiated thyroid cancer. Thyroid 2016 Jan;26(1):1-133 [FREE Full text] [doi: 10.1089/thy.2015.0020] [Medline: 26462967] 
10. Tuttle R, Haddad R. National Comprehensive Cancer Network. 2014. NCCN Clinical Practic Guidelines in Oncology: thyroid carcinoma. Version 2.2014 URL: https://www.nccn.org/patients/guidelines/content/PDF/thyroid-patient.pdf [accessed 2020-01-14]

11. Ryu J, Ryu YM, Jung Y, Kim S, Lee YJ, Lee E, et al. Extent of thyroidectomy affects vocal and throat functions: a prospective observational study of lobectomy versus total thyroidectomy. Surgery 2013 Sep;154(3):611-620. [doi: 10.1016/j.surg.2013.03.011] [Medline: 23932596]

12. Alexander C, Bader JB, Schaefer A, Finke C, Kirsch CM. Intermediate and long-term side effects of high-dose radioiodine therapy for thyroid carcinoma. J Nucl Med 1998 Sep;39(9):1551-1554 [FREE Full text] [Medline: 9744341]

13. Duan H, Gamper E, Becherer A, Hoffmann M. Quality of life aspects in the management of thyroid cancer. Oral Oncol 2015 Jun;51(6):S1-S5 [FREE Full text] [doi: 10.1016/j.oraloncology.2015.03.008] [Medline: 25920747]

14. Mihai LT, Milu C, Voicu B, Enachescu D. Ionizing radiation—understanding and acceptance. Health Phys 2005 Oct;89(4):375-382. [Medline: 16155459]

15. Williams A. Family caregivers to adults with cancer: the consequences of caring. Recent Results Cancer Res 2018;210:87-103. [doi: 10.1007/978-3-319-64310-6 6] [Medline: 28924681]

16. Tolbert E, Bowie J, Snyder C, Bantug E, Smith K. A qualitative exploration of the experiences, needs, and roles of caregivers during and after cancer treatment: "That's what I say. I'm a relative survivor”. J Cancer Surviv 2018 Dec;12(1):134-144. [doi: 10.1007/s11764-017-0652-x] [Medline: 29101710]

17. Duan H, Gamper E, Becherer A, Hoffmann M. Quality of life aspects in the management of thyroid cancer. Oral Oncol 2015 Jun;51(6):S1-S5 [FREE Full text] [doi: 10.1016/j.oraloncology.2015.03.008] [Medline: 25920747]

18. Stajduhar KI, Neithercut J, Chu E, Pham P, Rohde J, Sicotte A, et al. Thyroid cancer: patients' experiences of receiving iodine-131 therapy. Oncol Nurs Forum 2000 Sep;27(8):1213-1218. [Medline: 11013902]

19. Zhao J, Freeman B, Li M. Can mobile phone apps influence people's health behavior change? An evidence review. J Med Internet Res 2016 Oct 31;18(11):e287 [FREE Full text] [doi: 10.2196/jmir.5692] [Medline: 27806926]

20. Smahel D, Elavsky S, Machackova H. Functions of mHealth applications: a user's perspective. Health Informatics J 2017 Nov 01:1460458217740725. [doi: 10.1177/1460458217740725] [Medline: 29121831]

21. Coughlin SS. Introduction to focused issue on mHealth and social media interventions for cancer. Mhealth 2016;2:42 [FREE Full text] [doi: 10.21037/mhealth.2016.11.01] [Medline: 27917387]

22. US Food and Drug Administration. 2019 Sep 27. Policy for device software functions and mobile medical applications: guidance for industry and Food and Drug Administration staff URL: https://www.fda.gov/media/80958/download [accessed 2020-01-14]

23. Cortez NG, Cohen IG, Kesselheim AS. FDA regulation of mobile health technologies. N Engl J Med 2014 Jul 24;371(4):372-379. [doi: 10.1056/NEJMhle1403384] [Medline: 25054722]

24. Bender JL, Yue RYK, To MJ, Deacken L, Jadad AR. A lot of action, but not in the right direction: systematic review and content analysis of smartphone applications for the prevention, detection, and management of cancer. J Med Internet Res 2013;15(12):e287 [FREE Full text] [doi: 10.2196/jmir.2661] [Medline: 24366061]

25. Seiler A, Klaas V, Tröster G, Fagundes CP. eHealth and mHealth interventions in the treatment of fatigued cancer survivors: a systematic review and meta-analysis. Psychooncology 2017 Sep;26(9):1239-1253. [doi: 10.1002/pon.4489] [Medline: $\underline{28665554]}$

26. McCarroll ML, Armbruster S, Pohle-Krauza RJ, Lyzen AM, Min S, Nash DW, et al. Feasibility of a lifestyle intervention for overweight/obese endometrial and breast cancer survivors using an interactive mobile application. Gynecol Oncol 2015 Jun;137(3):508-515. [doi: 10.1016/j.ygyno.2014.12.025] [Medline: 25681782]

27. Hong YA, Goldberg D, Ory MG, Towne SD, Forjuoh SN, Kellstedt D, et al. Efficacy of a mobile-enabled web app (iCanFit) in promoting physical activity among older cancer survivors: a pilot study. JMIR Cancer 2015 Jun 26;1(1):e7 [FREE Full text] [doi: 10.2196/cancer.4389] [Medline: 28410158]

28. Odeh B, Kayyali R, Nabhani-Gebara S, Philip N. Optimizing cancer care through mobile health. Support Care Cancer 2015 Jul;23(7):2183-2188. [doi: 10.1007/s00520-015-2627-7] [Medline: 25649121]

29. Egbring M, Far E, Roos M, Dietrich M, Brauchbar M, Kullak-Ublick GA, et al. A mobile app to stabilize daily functional activity of breast cancer patients in collaboration with the physician: a randomized controlled clinical trial. J Med Internet Res 2016 Sep 06;18(9):e238 [FREE Full text] [doi: 10.2196/jmir.6414] [Medline: 27601354]

30. Nasi G, Cucciniello M, Guerrazzi C. The performance of mHealth in cancer supportive care: a research agenda. J Med Internet Res 2015 Feb 13;17(1):e9 [FREE Full text] [doi: 10.2196/jmir.3764] [Medline: 25720295]

31. Huang A, Chen C, Bian K, Duan X, Chen M, Gao H, et al. WE-CARE: an intelligent mobile telecardiology system to enable mHealth applications. IEEE J Biomed Health Inform 2014 Mar;18(2):693-702. [doi: 10.1109/JBHI.2013.2279136] [Medline: 24608067]

32. Singer S, Husson O, Tomaszewska IM, Locati LD, Kiyota N, Scheidemann-Wesp U, et al. Quality-of-life priorities in patients with thyroid cancer: a multinational European organisation for research and treatment of cancer phase I study. Thyroid 2016 Dec;26(11):1605-1613. [doi: 10.1089/thy.2015.0640] [Medline: 27605136] 
33. Johnson C, Aaronson N, Blazeby J, Bottomley A, Fayers P, Koller M. European Organisation for Research and Treatment of Cancer. 2011 Apr. Guidelines for developing questionnaire modules URL: http://groups.eortc.be/qol/sites/default/files/ archives/guidelines for developing questionnaire- final.pdf [accessed 2020-01-14]

34. Singer S, Jordan S, Locati LD, Pinto M, Tomaszewska IM, Araújo C, EORTC Quality of Life Group, EORTC Head and Neck Cancer Group, EORTC Endocrine Task Force. The EORTC module for quality of life in patients with thyroid cancer: phase III. Endocr Relat Cancer 2017 Dec;24(4):197-207. [doi: 10.1530/ERC-16-0530] [Medline: 28223365]

35. Singer S, Al-Ibraheem A, Guy A. European Organisation for Research and Treatment of Cancer. QOL module for thyroid cancer QLQ-THY34 URL: http://groups.eortc.be/qol/thyroid-cancer-qlq-thy34 [accessed 2020-01-14]

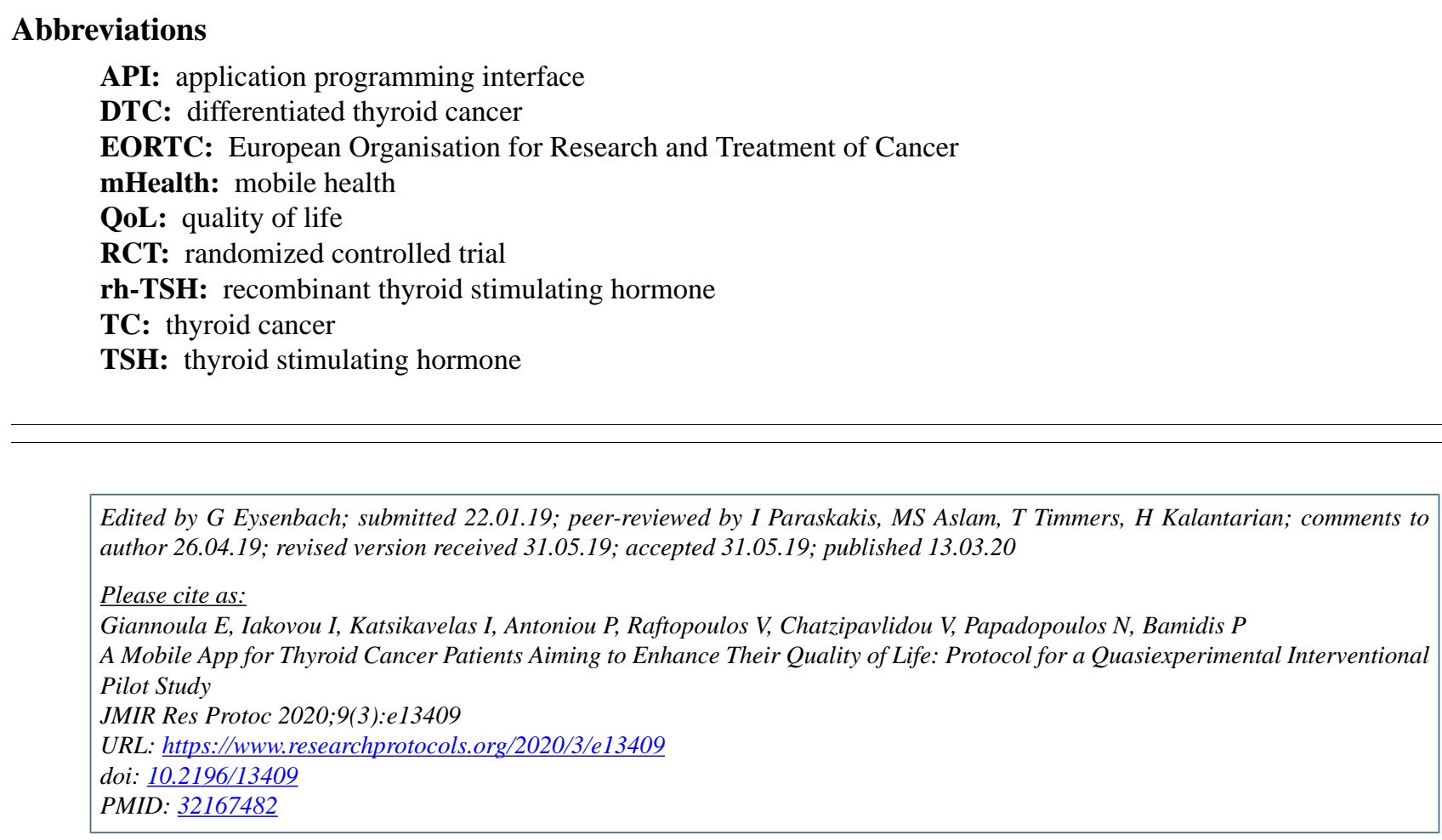

(CEvanthia Giannoula, Ioannis Iakovou, Ioannis Katsikavelas, Panagiotis Antoniou, Vasilios Raftopoulos, Vasiliki Chatzipavlidou, Nikitas Papadopoulos, Panagiotis Bamidis. Originally published in JMIR Research Protocols (http://www.researchprotocols.org), 13.03.2020. This is an open-access article distributed under the terms of the Creative Commons Attribution License (https://creativecommons.org/licenses/by/4.0/), which permits unrestricted use, distribution, and reproduction in any medium, provided the original work, first published in JMIR Research Protocols, is properly cited. The complete bibliographic information, a link to the original publication on http://www.researchprotocols.org, as well as this copyright and license information must be included. 